\title{
Anti-inflammatory potential of an essential oil-containing mouthwash in elderly subjects enrolled in supportive periodontal therapy: a 6-week randomised controlled clinical trial
}

\author{
Hendrik Jünger ${ }^{1}$ - Anna Jaun-Ventrice ${ }^{1} \cdot$ Kevin Guldener $^{2} \cdot$ Christoph A. Ramseier $^{2}$ - Daniel R. Reissmann ${ }^{3}$. \\ Martin Schimmel ${ }^{1,4}$ (iD
}

Received: 20 October 2019 / Accepted: 27 December 2019 / Published online: 8 January 2020

(C) The Author(s) 2020

\begin{abstract}
Objectives Essential oils and other plant extracts have evoked interest as sources of natural medicinal products. They have been proven to exert antibacterial, antifungal, antiviral and antioxidant properties, but the mechanism of action has not been fully elucidated.

Aim This study aims to evaluate the potential of a sage-containing mouthwash to alleviate inflammatory signs of intra-oral mucosa and gingiva in comparison with a water/alcohol-based placebo.

Material and methods This study was conducted as a randomised, placebo-controlled, double-blind, parallel design clinical study. Forty-eight dentate subjects were randomly assigned to a test (sage-containing mouthwash) or a placebo group (water/ alcohol-based solution). Subjects rinsed once daily for 30 s over a period of 6 weeks. Sulcus Bleeding Index (SBI), Plaque Index (PLI), tooth staining, xerostomia and degree of stomatitis were assessed at baseline and after 6 weeks.

Results Subjects' mean age was 77.5 \pm 7.3 years. SBI was reduced from $1.3 \pm 0.9$ to $0.8 \pm 0.7$ (test, $p=0.0029$ ) and $1.4 \pm 0.9$ to $1.1 \pm 0.7$ (placebo, $p=0.0105$ ). Similarly, PLI was reduced from $1.2 \pm 0.5$ to $1.0 \pm 0.3$ (test, $p=0.0080$ ) and $1.3 \pm 0.4$ to $1.1 \pm 0.6$ (placebo, $p=$ 0.0087); no between-group differences were found $(p>0.05)$. Stomatitis, xerostomia and tooth staining revealed no change after 6 weeks. Conclusion The irrigation with a sage-containing mouthwash did not result in a superior beneficial effect on inflammatory parameters and plaque indices compared with the placebo. The expected contribution of the plant extracts to their potential impact on oral health may need further investigation.

Clinical relevance The proposed mouthwash might be suitable for patients with inflammatory signs of the gingiva who prefer natural remedies.
\end{abstract}

Keywords RCT $\cdot$ Gingival inflammation $\cdot$ Mouthwash $\cdot$ Essential oils $\cdot$ Oral hygiene $\cdot$ Mucosa

Electronic supplementary material The online version of this article (https://doi.org/10.1007/s00784-019-03194-3) contains supplementary material, which is available to authorized users.

Martin Schimmel

martin.schimmel@zmk.unibe.ch

1 Division of Gerodontology, Department of Reconstructive Dentistry and Gerodontology, School of Dental Medicine, University of Bern, Freiburgstrasse 7, 3010 Bern, Switzerland

2 Department of Periodontology, University of Bern, Bern, Switzerland

3 Department of Prosthetic Dentistry, University Medical Center Hamburg-Eppendorf, Hamburg, Germany

4 Division of Gerodontology and Removable Prosthodontics, University of Geneva, Geneva, Switzerland

\section{Introduction}

A greater number of people today retain their natural teeth until later in life. For example, in Switzerland, only $6.5 \%$ of the population above the age of 65 years are reported to be edentulous [1]. Overall, the incidence of edentulism is declining due to the success of both preventive and conservative dentistry [2] as well as restorative dental techniques [3]. Current assessments from Germany, however, indicate that despite these preventive efforts, the prevalence of oral infections is still increasing with age. Later in life, periodontal disease is commonly found to be the main reason for tooth loss [4].

In elderly patients who are in need of help for their everyday activities (activities of daily living (ADL)) both the oral and denture hygiene is often poor $[5,6]$. Thus, more 
frequently, periodontal inflammation may be leading to pain, tooth loss, decreased masticatory function and reduced quality of life $[7,8]$. Furthermore, it has been shown that inappropriate oral health may further compromise general health especially in elderly patients. In these cases, the accumulated biofilm built on teeth and dentures can be released into saliva, the oropharynx and further be aspirated into the lungs possibly causing aspiration pneumonia [9]. A number of studies suggested that aspiration pneumonia is an important risk factor for morbidity and mortality in elderly patients $[10,11]$. On the other hand, Yoneyama and co-workers conducted a study in nursing homes demonstrating that rigorous oral hygiene regimes may result in a significant risk reduction for the development of aspiration pneumonia [12].

The potential pathogens such as Staphylococcus aureus can be detected in $20 \%$ and enterobacteria in $8 \%$ of the elderly population. Furthermore, yeasts are highly prevalent in people living in long-term care facilities [13]. They form biofilms on teeth, mucosa and denture surfaces, which could most commonly be removed mechanically using a toothbrush and toothpaste. However, many elderly people are not able to perform a homecare oral hygiene regimen as indicated. They often lack the capacity for adequate oral hygiene due to decreasing manual dexterity, impaired vision or frailty [14].

Several indications have been described in which antimicrobial mouthwashes may be of additional value to mechanical plaque control. These clinical situations may comprise poor mechanical control (e.g. arthritis of the hands), xerostomia, use of splints, fixed partial prostheses, exposed root surfaces, overdenture abutments or oral surgery [15]. In such cases, antimicrobial mouthwashes may add a significant benefit, especially chlorhexidine in concentrations of 0.1 to $0.2 \%$ [16]. Other mouthwashes containing fluoride were shown to exhibit anti-inflammatory effects, however, demonstrating common side effects such as mouth-burning sensations and staining of both teeth and tongue [17].

Essential oils and other plant extracts have evoked interest as a source of natural medicinal products. Both oils and plant extracts have been evaluated for their use as an alternative medicine in the treatment of numerous infectious diseases [18]. Specifically, essential oils were proven to exert antibacterial, antifungal, antiviral and antioxidant properties. However, their mechanisms of action have not been fully elucidated [19]. Salvia officinalis (sage) has been used in medicine and in dentistry for several hundred years. A recent study demonstrated an anticandidal effect of Salvia officinalis essential oil against Candida albicans [20]. Delamare and co-workers [21] demonstrated an antibacterial activity of the essential oils of Salvia officinalis and Salvia triloba. The essential oils of both species exhibited bacteriostatic properties against several microorganisms, among them Staphylococcus aureus [21].

Further information on the effect of sage-containing mouthwashes on periodontal health may lead to improved management of periodontal diseases in elderly individuals enrolled in supportive periodontal therapy (SPT). Therefore, the main aim of the current study was to evaluate the potential of a sage-containing mouthwash to reduce inflammatory signs of both intra-oral mucosa and gingiva in comparison with a water/alcohol-based placebo.

Null-hypothesis HO: In elderly patients under supportive periodontal therapy, there is no difference between a sagecontaining mouthwash and a water/alcohol-based, tasteadjusted placebo mouthwash in alleviating inflammatory signs of intra-oral mucosa and gingiva.

\section{Material and methods}

\section{Subjects, study design and setting}

This was a randomised, placebo-controlled, doubleblind, parallel design clinical study. The consolidation standards of reporting trials (CONSORT) guidelines for clinical trials were followed [22]. The study was reviewed, registered and approved by the appropriate Regional Ethical Board in Bern, Switzerland (BasecNo.: 2016-01383). The study was registered on the ClinicalTrials.gov Protocol Registration and Results System (PRS) under the ID: NCT0283080. The recruitment period lasted from 2016 to 2017. Consecutive patients from a private geriatric practice (Laupen, Switzerland), two homes for the elders (Burgerspittel am Viererfeld, Bern and Betagtenzentrum Laupen) and the School of Dental Medicine (Bern, Switzerland) were informed about the study and asked whether they wanted to participate. Inclusion criteria were the ability to give informed consent and the willingness to participate in the study. Subjects needed to be 65 years and older while presenting a full-mouth percentage of bleeding on probing $(\mathrm{BOP})>50 \%$ [23]. Exclusion criteria were allergy to one of the components of the irrigation solution, complete edentulism, alcohol dependency, prescription of antibiotics or immunosuppressant (e.g. glucocorticoids) and anti-inflammatory mouthwash. There was no minimum time period that must have elapsed from the last recall for the patients to be included in the study. One calibrated dentist, who was blinded to the type of mouthwash investigated in this study, conducted all clinical examinations. Measurements were recorded on case report forms (CRF) specifically designed for this study. For all subjects, the baseline examination (BL) included an independent oral examination together with the collection of the general medical history.

A priori sample size calculation could not be performed due to lack of previous reference data. Therefore, a sample size of $n=40$ was chosen and a recruitment of $n=48$ was 
deemed necessary to compensate for dropouts. This study was designed as a pilot randomised clinical trial (RCT).

Randomisation was performed according to an onlinegenerated randomisation list (www.randomizer.org) with blocs of $n=8$. Attribution to either the test group or the placebo group was noted and concealed in non-transparent envelopes. Following initial screening, signing of informed consent and study inclusion as well as the baseline examination by the calibrated dentist, the envelope was opened and the subject was thus attributed to the test group or the placebo group, respectively.

\section{Intervention}

For the duration of the study, the test group rinsed with a sagecontaining mouthwash (Dr. Hauschka Med Mundspülung Salbei, WALA Heilmittel GmbH, Bad Boll/Eckwälden, Germany) containing extraction of Althaea officinalis leaf extract, Salvia officinalis leaf extract, Krameria Triandra root extract, Calendula officinalis flower extract, Potentilla erecta root extract, Aesculus hippocastanum bark extract, Melia azadirachta leaf extract, Commiphora Myrrha resin extract, water and alcohol solvent (9 vol\%). The placebo group rinsed with a placebo mouthwash (WALA Heilmittel $\mathrm{GmbH}$ ) that was similar in taste, colour and alcohol content (9 vol\%). Stability tests were performed for all mouthwashes used, and a safety assessment was conducted according to the EU Cosmetic Law $1223 / 2009$. Both test and placebo mouthwashes were packed in identical white bottles which were labelled with consecutive numbers only. Hence, both operator and subjects were blinded to the assignment of the study group.

In addition to their individual oral hygiene measures, the subjects were asked to rinse with the allocated mouthwash once daily (in the evening after brushing or before bedtime) for the duration of 6 weeks. Patients were instructed to rinse vigorously for $30 \mathrm{~s}$. They have not been given instructions to improve oral hygiene. For the standardisation of the time used for rinsing, an hourglass was provided. After rinsing with the mouthwash, participants were advised to avoid further rinsing, eating or drinking for at least $30 \mathrm{~min}$.

\section{Primary outcome measure}

For the purpose of this study, the Sulcus Bleeding Index (SBI) was used as the primary outcome measure [24]. The criteria for scoring were Score 0 (healthy gingiva and absence of bleeding on probing (BOP)), Score 1 (healthy gingiva and presence of $\mathrm{BOP}$ ), Score 2 (change in colour of the gingiva, no oedema, presence of BOP), Score 3 (change in colour of the gingiva, slight oedema, presence of BOP), Score 4 (change in colour of the gingiva, obvious oedema, presence of BOP) and Score 5 (change in colour of the gingiva, marked oedema, spontaneous bleeding). Four gingival sites were scored at each tooth: mid-buccal, mid-lingual and the mesio-buccal and disto-buccal papillary gingiva. The average scores recorded determined the SBI.

\section{Secondary outcome measures}

\section{Plaque Index}

Plaque deposits were recorded using the Plaque Index (PLI) according to Löe and Silness: Score 0 (absence of plaque), Score 1 (plaque disclosed after running the periodontal probe along the gingival margin), Score 2 (visible plaque) and Score 3 (abundance of plaque) [25].

\section{Tooth Staining Index}

The vestibular and oral surfaces were evaluated for discolouration, ignoring wisdom teeth, crowned teeth and dentures using the Tooth Staining Index (TSI) [17]. The staining was graded: Grade 0 (no discolouration), Grade 1 (up to $1 /$ 4 of the tooth surface was discoloured), Grade 2 (up to $1 / 2$ of the tooth surface was discoloured), Grade 3 (up to $3 / 4$ of the tooth surface was discoloured) and Grade 4 (more than 3/4 of the tooth surface was discoloured). The TSI was calculated as the sum of all discolouration areas over the sum of all surfaces.

\section{Xerostomia}

Subjective symptoms of dry mouth were assessed using the German 14-item version of the Xerostomia Inventory (XI) [26]. Subjects were asked to indicate the frequency of 14 occurrences that are related to dry mouth and throat using a 5-point ordinal rating scale. The XI summary score is the sum of the scores of the 14 single items with potential values ranging from 0 to 56 and with higher values indicating more symptoms of dry mouth. Therefore, the XI was considered a problem index. Reliability of the XI was assessed as the instrument's internal consistency.

\section{Stomatitis Index}

Oral soft tissues were further assessed using the Stomatitis Index (SI) according to Newton [27] as follows: Grade 0 (none), Grade 1 (punctate hyperaemic lesions, local erythema), Grade 2 (diffuse erythema, generalised simple inflammation) and Grade 3 (hyperplastic granular surface, inflammatory papillary hyperplasia) [27].

\section{Statistical analysis}

A descriptive analysis was conducted, and absolute and relative frequencies, mean values and standard deviations were 
reported. The data were evaluated using the statistical software package R, Release 3.3.3 [28].

With the perceived xerostomia, a Cronbach's alpha [29] was computed along with the average inter-item correlation using all $14 \mathrm{XI}$ items. Alpha values were interpreted according to guidelines with values of 0.70 or above suggesting satisfactory reliability [30]. For broader higher-order constructs such as dry mouth symptoms, an average inter-item correlation of at least 0.15 was considered satisfactory [31]. Cronbach's alpha $(0.90)$ and average inter-item correlation $(0.39)$ revealed that the instrument's internal consistency was excellent.

The baseline results of both groups were tested using Students $t$ test for independent samples and Fisher's exact test. Differences between baseline and reexamination were tested using both Student's paired $t$ test and Fisher's exact test. Both tests were corrected for repeated measurements according to the method described by Holm. For the prediction of the difference of numeric values between the baseline and the follow-up examination, multiple linear regression models were used. The explanatory variable 'tooth brushing frequency' and their response options 'once, occasionally or never' were combined in one group as opposed to the frequency 'twice or more'. As level of significance, a $p$ value of $<0.05$ was chosen.

Fig. 1 The CONSORT 2010 flow diagram of the present study

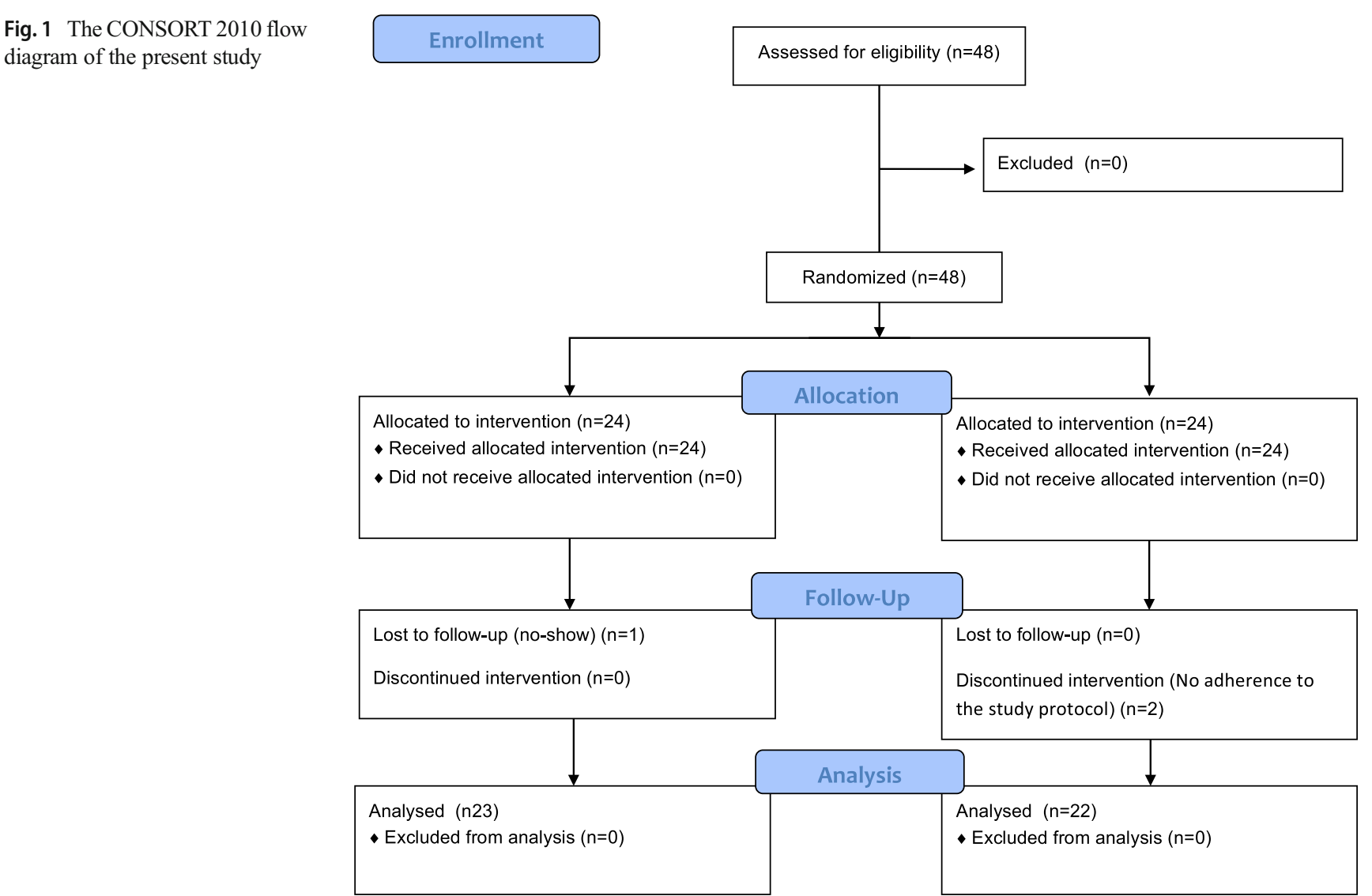

\section{Results}

\section{Subjects}

Out of 48 initially included subjects, 45 completed the study (test $n=23$, control $n=22$, Fig. 1). Reasons for dropout were no compliance to the study protocol $(n=2)$ or no show at the final examination $(n=1)$. The mean age of the subjects was 77.5 years $( \pm 7.3)$, and the majority were non-smokers (83.3\%). Out of the $n=48$ subjects, $52.1 \%(n=25)$ were females. The mean number of drugs taken by the study participants was $4.6( \pm 5.16, \min =0, \max =19)$, and none of them was diabetic. In the control group, 14 participants brushed their teeth at least twice a day and 10 once per day. In the test group, 19 participants brushed their teeth at least twice a day, another 4 once per day and 1 occasionally. The difference was not significant $(p=0.270)$. Baseline and demographic characteristics were not statistically significantly different between the two study groups (Table 1).

\section{Outcome measures}

Gingival inflammation as assessed by the SBI in the test group improved from $1.3( \pm 0.9)$ to $0.8( \pm 0.7)(p=0.0029)$ and 1.4 $( \pm 0.9)$ to $1.1( \pm 0.7)(p=0.0105)$ in the placebo group, 
Table 1 Baseline demographic data and unadjusted qualitative analysis of possible confounders in $n=48$ subjects

\begin{tabular}{lllll}
\hline & Placebo $(n=24)$ & Test $(n=24)$ & All $(n=48)$ & $p$ value \\
\hline Age (years) & $76.2 \pm 6.5$ & $78.7 \pm 7.9$ & $77.5 \pm 7.3$ & 0.2376 \\
Sex & $12(50.0 \%)$ women & $13(54.2 \%)$ women & $25(52.1 \%)$ women & 1.0000 \\
& $12(50.0 \%)$ men & $11(45.8 \%)$ men & $23(47.9 \%)$ men & \\
Existing teeth $(n)$ & $17.5 \pm 5.9$ & $16.9 \pm 7.9$ & $17.2 \pm 6.9$ & 0.7577 \\
DMFT $(n)$ & $22.1 \pm 3.6$ & $24.0 \pm 3.9$ & $22.1 \pm 5.8$ & 0.0994 \\
Smoker & & & & \\
No & $21(87.5 \%)$ & $19(79.2 \%)$ & $40(83.3 \%)$ & 0.8290 \\
Yes & $2(8.3 \%)$ & $4(16.7 \%)$ & $6(12.5 \%)$ & \\
Unknown & $1(4.2 \%)$ & $1(4.2 \%)$ & $2(4.2 \%)$ & \\
Alcohol & & & & 1.0000 \\
No & $6(25.0 \%)$ & $7(29.2 \%)$ & $33(68.8 \%)$ & \\
Yes & $17(70.8 \%)$ & $16(66.7 \%)$ & $2(4.2 \%)$ & \\
Unknown & $1(4.2 \%)$ & $1(4.2 \%)$ & & \\
\hline
\end{tabular}

Five patients (four in the test group, one in the control group) were restored mixed, both on natural teeth and implants (total number of implants $n=20$ and $n=1209$ natural teeth in the study population)

$D M F T$, decayed missing filled teeth respectively. Furthermore, the calculated change of SBI for each site of measurement was not statistically significant between test and placebo groups (chi-square test, $p=0.8823$ ). Similarly, the PLI in the test group improved from $1.2( \pm 0.5)$ to $1.0( \pm 0.3)(p=0.0080)$ and $1.3( \pm 0.4)$ to $1.1( \pm 0.6)(p=$ 0.0087 ) in the placebo group, respectively (Fig. 2). No statistically significant differences were found, however, between test and placebo groups at both baseline and the 6-week follow-up ( $p>0.05$ ) (Fig. 2; Supplementary Table A).

There was no statistically significant difference in the pooled analysis concerning the degree of prosthetic stomatitis at baseline $(p=0.6824)$ and follow-up $(p=0.2362)$, tooth staining in both upper and lower jaw (baseline and followup: $p>0.05$ ) and xerostomia at baseline and follow-up and no difference between groups $(p>0.05)$, respectively (Table 2).

\section{$\mathrm{Bi}$ - and multivariate analyses}

The linear regression analysis of the intervention effect revealed that either increase or decrease of SBI and PLI could not be predicted from the groups. There was a tendency, however, that the improvement in PLI could be predicted from age since younger subjects tended to show a more improved PLI (Table 3).

\section{Discussion}

\section{Summary of the results}

During the course of the study, periodontal indices related to gingival health and oral hygiene improved in both study groups reaching statistical significance. However, no between-group differences were found among test and placebo nor could the effect of the test group be predicted from the statistical model for age, gender, frequency of tooth brushing, and the allocation of the study group. Therefore, the nullhypothesis $\mathrm{H} 0$ cannot be rejected. However, it has to be noted that the study revealed an effect size for the improvement of the main outcome parameter gingival inflammation as assessed with the SBI of 0.42 . An effect size of 0.5 was classified as 'medium' and could be 'visible to the naked eye of a careful observer' according to Sullivan and Feinn [32]. Hence, it can be assumed that the current study was slightly underpowered.

\section{Results in the context of existing knowledge}

The effects of plant extracts and essential oils have attracted considerable interest, but most of these studies had been performed in vitro [33]. They described bacteriostatic and antifungal effects and alteration to the morphology of the bacterial organisms. The present study revealed that both the placebo solution and the sage-containing mouthwash had a similar effect on gingival inflammatory parameters. It has to be noted, however, that the placebo solution possibly contained effective agents in the same concentration such as alcohol. Therefore, a possible beneficial effect of the alcohol and peppermint oil content of the placebo on the composition of the intraoral flora could be taken into consideration. However, it has to be noted that Chalhoub and co-workers in their RCT did not find a beneficial effect of using an alcohol-free essential oil-containing mouthwash containing peppermint oil in institutionalised patients with dementia [34]. 
Fig. 2 Mean Sulcus Bleeding Index (SBI) and Plaque Index (PLI) from $n=23$ test subjects and $n=22$ control subjects at baseline and 6 weeks. $* p<0.05$, statistically significant difference
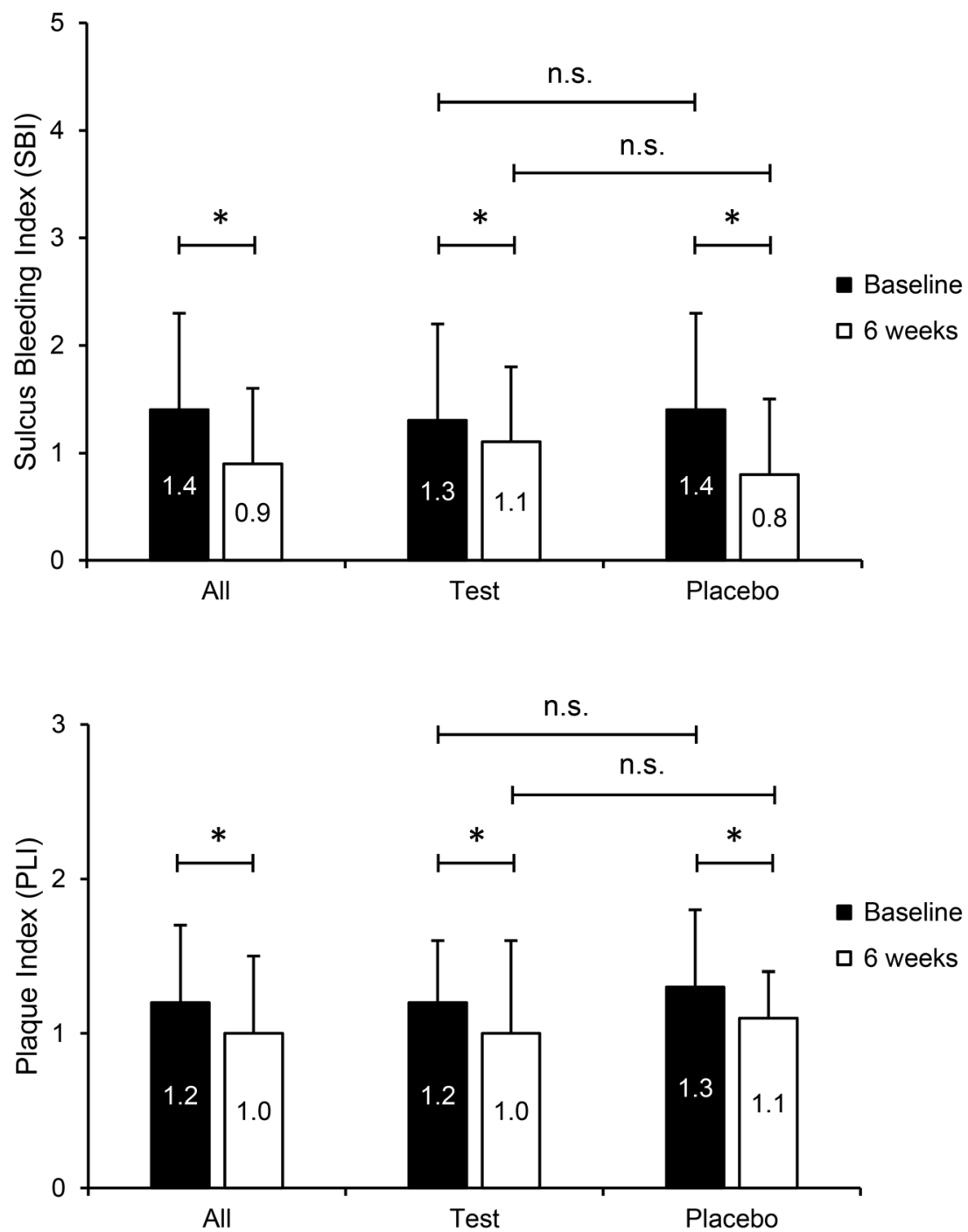

In an in vitro study, Sissons and co-workers demonstrated that prolonged application of ethanol of up to $40 \%$ is necessary to inhibit growth of plaque biofilms [35]. With an alcohol concentration as solvent of 9 vol\%, both test and control solutions of the present study were not expected to have a statistically significant inhibitory effect on the microbial flora. In a 3-day plaque regrowth model Marchetti and co-workers found no difference between an alcohol-free mouthwash containing essential oils compared with an alcohol-based essential oil mouthwash [36]. The lack of a placebo group rinsing with a non-alcohol-containing placebo may be considered a limitation of the present study.

In a systematic review, van Leeuwen and co-authors analysed the effect of an alcohol vehicle solution compared with an essential oil mouthwash and a water-based control [37]. The evidence from their review revealed that the reduction in plaque and gingivitis between the alcohol vehicle solution and the water control was not significantly different.
Therefore, it may be concluded that the antiseptic effect of the hydro-alcohol solution appears to be negligible [37].

One further possible explanation of the improvement in both SBI and PLI may be attributed to the Hawthorne effect. More specifically, the improvement of the PLI over 6 weeks highly reaching statistical significance supports this assumption. The Hawthorne effect, i.e. 'the observer effect' was described as the reaction of individuals of being observed and consequently, change their behaviour because of the circumstance of being observed. It was suggested that the knowledge of being subject to research, and subsequent higher attention to the individual would lead to an increase in productivity or observed action [38]. The awareness of being studied in the present RCT may have had a positive impact on the oral hygiene behaviour of the subjects in our control group. Such consequences have been repeatedly described, but very little is known about the mechanism under which they operate [39].

Another RCT by Bellomo and co-workers [40] assessed the improvement of oral hygiene measures for elderly people 
Table 2 Descriptive summary of the degree of stomatitis, tooth staining and xerostomia inventory per group and clinical visit

\begin{tabular}{lllll}
\hline & All & Placebo & Test & $p$ values \\
\hline Prosthetic stomatitis & & & & \\
BL $(n=48)$ & & & & \\
None & $7(14.6 \%)$ & $3(12.5 \%)$ & $4(16.7 \%)$ & 0.6824 \\
Localised & $14(29.2 \%)$ & $6(25.0 \%)$ & $8(33.3 \%)$ & \\
Generalised simple & $7(14.6 \%)$ & $5(20.8 \%)$ & $2(8.3 \%)$ & \\
NA & $20(41.7 \%)$ & $10(41.7 \%)$ & $10(41.7 \%)$ & \\
6 W (n=45) & & & & \\
None & $7(14.6 \%)$ & $3(12.5 \%)$ & $4(16.7 \%)$ & 0.2362 \\
Localised & $13(27.1 \%)$ & $4(16.7 \%)$ & $9(37.5 \%)$ & \\
Generalised simple & $2(4.2 \%)$ & $2(8.3 \%)$ & $0(0 \%)$ & \\
NA (dropout) & $23(47.9 \%)$ & $13(54.2 \%)$ & $10(41.7 \%)$ & \\
& $3(6.3 \%)$ & $2(8.3 \%)$ & $1(4.3 \%)$ & \\
Tooth Staining Index & & & & \\
Upper jaw & & & & \\
BL $(n=48)$ & $1.0 \pm 0.6$ & $1.0 \pm 0.5$ & $1.0 \pm 0.7$ & 0.9643 \\
6 W ( $n=45)$ & $0.8 \pm 0.6$ & $0.8 \pm 0.6$ & $0.7 \pm 0.5$ & 0.5139 \\
Lower jaw & & & & \\
BL $(n=48)$ & $1.3 \pm 0.5$ & $1.3 \pm 0.5$ & $1.2 \pm 0.5$ & 0.4120 \\
6 W $(n=45)$ & $0.9 \pm 0.5$ & $1.0 \pm 0.6$ & $0.9 \pm 0.6$ & 0.5356 \\
Xerostomia Inventory & & & & \\
BL $(n=48)$ & $8.5 \pm 8.7$ & $7.7 \pm 6.2$ & $8.9 \pm 11.4$ & 0.6602 \\
6 W $(n=45)$ & $8.25 \pm 10.0$ & $8.2 \pm 10.4$ & $10.7 \pm 10.0$ & 0.4105 \\
\hline
\end{tabular}

$B L$, baseline; $6 \mathrm{~W}, 6$-week follow-up

in a long-term care facility. Their experimental group benefitted from ergo-therapy for the improvement of the autonomously performed oral hygiene. Their control group received regular manicure. Interestingly, in both groups, oral and denture plaque scores improved [40]. Their observation may also suggest a similar psychological effect observed in the present study.

Table 3 Regression analysis for the Sulcus Bleeding Index (SBI) and further indicators of gingival health

\begin{tabular}{lccc}
\hline & Beta $(95 \% \mathrm{CI})$ & SE & $p$ value \\
\hline & & & \\
\multicolumn{4}{l}{ Sulcus Bleeding Index (SBI) } \\
Group (test vs. placebo) & $-0.05(-0.47,0.37)$ & 0.2061 & 0.7437 \\
Gender & $0.15(-0.28,0.52)$ & 0.1983 & 0.5937 \\
Age & $0.004(-0.023,0.032)$ & 0.0135 & 0.7316 \\
Tooth brushing frequency & $-0.13(-0.57,0.31)$ & 0.2174 & 0.5527 \\
Plaque Index (PLI) & & & \\
Group (test vs. placebo) & $-0.03(-0.22,0.15)$ & 0.0908 & 0.8833 \\
Gender & $-0.03(-0.21,0.14)$ & 0.0874 & 0.7723 \\
Age & $0.01(-0.00,0.02)$ & 0.0060 & 0.0787 \\
Tooth brushing frequency & $0.06(-0.13,0.26)$ & 0.0958 & 0.5202 \\
\hline
\end{tabular}

SE, standard error
The influence of alcohol containing mouthwashes on the oral mucosa is repeatedly discussed in the medical and dental literature. The effect of alcohol on keratinocytes as the most common cell type has been recently investigated in an in vitro study by Calderòn-Montāno and co-workers [41]. After exposition of keratinocytes to ethanol concentrations commonly used in mouthwashes, a marked cytotoxic effect was observed for concentrations of $20 \%$ and above. Cytotoxicity appears to be influenced by ethanol concentration and exposure time. Interestingly enough, no cytotoxicity was noted when cells were exposed to ethanol $10 \%$ for $5 \mathrm{~min}$. Therefore, we could not expect that the test and placebo solution with a concentration of 9 vol\% alcohol to cause any adverse effects on the oral mucosa.

Boffetta and co-workers pointed out that most of the available mouthwashes contain 20\% alcohol and thus suggested a possible relationship with oral cancer [42]. Due to many confounding factors, however, conclusive evidence is lacking. Based on the risk estimation of three to four cases per one million, it will hardly be possible to epidemiologically detect and determine the level of risk from alcohol containing mouthwashes on oral cancer [43].

For the evaluation of xerostomia, the German adaption of the XI score according to Thomson and coworkers has been evaluated in the current study [26]. These authors have shown a change of 6 or more points in the XI score being clinically meaningful. We did not find a worsening effect on xerostomia (point difference, 0.5-1.8), despite that placebo and test solution were containing alcohol. This is in agreement with the data of Nair and co-workers who evaluated the 7-day rinsing effect of a $20 \%$ alcohol-containing mouthwash versus mouthwash without alcohol [44]. In contrast with our data, their patients were rinsing two times a day for $30 \mathrm{~s}$. We conclude that even long-term use of alcohol containing concentration $<10 \%$ mouthwashes are not worsening xerostomia.

\section{Strengths and weaknesses of the study}

This study was designed and conducted as a randomised, placebo-controlled, double-blind, parallel design clinical study. Hence, it fulfils the highest standards of clinical trial designs. If the study had been designed as a case series insteadwithout placebo control - the results would have appeared much more positive for the tested mouthwash, i.e. significant improvement of the inflammatory signs.

Taking the medium effect size of 0.42 for the SBI into consideration, it has to be extrapolated that the study was slightly underpowered. Although hypothetical, it is likely that a statistically significant effect could have been demonstrated if the sample size had been chosen to be higher. However, RCTs are very expensive and laborious studies and recruitment must be planned a priori. 
The compliance of the participants to the study protocol was impossible to supervise on a daily basis; however, at the end of the study, the filling level of the three bottles was checked.

A further strength of this study is that it was mainly practice-based and aimed at including old and very old dentate patients. Practice-based studies reflect much more dentists and their patients in normal life. Often, University-based studies lack the authenticity as diagnosis and treatment do not depend on financial and time constraints.

\section{Clinical implications}

Mouthwashes used on a regular basis appear to positively influence gingival health of elderly people. Participation in a study may account for an additional increase in the patient's motivation and awareness of oral hygiene measures supporting the importance of repeated patient motivation.

\section{Suggestions for further research}

In future trials evaluating sage-containing mouthwashes, it may be beneficial to add either a negative control group irrigating with a placebo solution not containing alcohol or a positive control group rinsing with chlorhexidine digluconate. Moreover, an irrigation time of 1 min twice per day may show more favourable results. Additionally, the follow-up time may be extended to two and 3 months in order to evaluate whether the expected improvement of the selected outcome measures may be demonstrated both mid- and long term. The setting of this type of trial including subjects in long-term care would be of additional interest. Several attempts have been made to improve oral hygiene in care settings; however, study results remained inconclusive.

\section{Conclusion}

In this study, the irrigation with a sage-containing mouthwash did not result in a superior beneficial effect on inflammatory parameters and plaque indices compared with the placebo. The potential impact of this plant extract on oral health may need further investigation.

Funding information This study was initiated and funded by WALA Heilmittel GmbH, Dorfstraße 1, 73,087 Bad Boll/Eckwälden, Germany. The Division of Gerodontology, University of Bern, received the funds. They were handled by the financial department, University of Bern, Switzerland. The authors of this study did not receive any cash or inkind benefits.

\section{Compliance with ethical standards}

Conflict of interest The authors declare that they have no conflict of interest.
Ethical approval All procedures performed in studies involving human participants were in accordance with the ethical standards of the institutional and/or national research committee and with the 1964 Helsinki declaration and its later amendments or comparable ethical standards. The study was reviewed, registered and approved by the appropriate Regional Ethical Board in Bern, Switzerland (Basec-No.: 2016-01383). The study was registered on the ClinicalTrials.gov Protocol Registration and Results System (PRS) under the ID: NCT0283080.

Informed consent Informed consent was obtained from all individual participants included in this study. They received oral and written information about the design of the study and their right to withdraw at any time without negative effect on their treatment.

Open Access This article is licensed under a Creative Commons Attribution 4.0 International License, which permits use, sharing, adaptation, distribution and reproduction in any medium or format, as long as you give appropriate credit to the original author(s) and the source, provide a link to the Creative Commons licence, and indicate if changes were made. The images or other third party material in this article are included in the article's Creative Commons licence, unless indicated otherwise in a credit line to the material. If material is not included in the article's Creative Commons licence and your intended use is not permitted by statutory regulation or exceeds the permitted use, you will need to obtain permission directly from the copyright holder. To view a copy of this licence, visit http://creativecommons.org/licenses/by/4.0/.

\section{References}

1. Schneider C, Zemp E, Zitzmann NU (2017) Oral health improvements in Switzerland over 20 years. Eur J Oral Sci 125:55-62. https://doi.org/10.1111/eos.12327

2. Sculean A, Bastendorf KD, Becker C, Bush B, Einwag J, Lanoway C, Platzer U, Schmage P, Schoeneich B, Walter C, Wennstrom JL, Flemmig TF (2013) A paradigm shift in mechanical biofilm management? Subgingival air polishing: a new way to improve mechanical biofilm management in the dental practice. Quintessence Int 44:475-477. https://doi.org/10.3290/j.qi.a29615

3. Holm-Pedersen P, Lang NP, Muller F (2007) What are the longevities of teeth and oral implants? Clin Oral Implants Res 18(Suppl 3): 15-19. https://doi.org/10.1111/j.1600-0501.2007.01434.x

4. Jordan AR, Micheelis W (2016) Fünfte Deutsche Mundgesundheitsstudie (DMS V). Fifth German Oral Health Survey. Deutscher Zahnärzteverlag Köln, Cologne

5. Katsoulis J, Schimmel M, Avrampou M, Stuck AE, Mericske-Stern $R$ (2012) Oral and general health status in patients treated in a dental consultation clinic of a geriatric ward in Bern, Switzerland. Gerodontology 29:e602-e610

6. Hoben M, Clarke A, Huynh KT, Kobagi N, Kent A, Hu H, Pereira RAC, Xiong T, Yu K, Xiang H, Yoon MN (2017) Barriers and facilitators in providing oral care to nursing home residents, from the perspective of care aides: a systematic review and meta-analysis. Int J Nurs Stud 73:34-51. https://doi.org/10.1016/j.ijnurstu. 2017.05.003

7. Schimmel M, Schoeni P, Zulian G, Müller F (2008) Utilisation of dental services in a university hospital palliative and long term care unit in Geneva. Gerodontology 25:107

8. Stenman U, Ahlqwist M, Bjorkelund C, Hakeberg M (2012) Oral health-related quality of life - associations with oral health and conditions in Swedish 70-year-old individuals. Gerodontology 29: e440-e446 
9. Sumi Y, Miura H, Sunakawa M, Michiwaki Y, Sakagami N (2002) Colonization of denture plaque by respiratory pathogens in dependent elderly. Gerodontology 19:25-29

10. Granton JT, Grossman RF (1993) Community-acquired pneumonia in the elderly patient. Clinical features, epidemiology, and treatment. Clin Chest Med 14:537-553

11. Niederman MS (1993) Nosocomial pneumonia in the elderly patient. Chronic care facility and hospital considerations. Clin Chest Med 14:479-490

12. Yoneyama T, Yoshida M, Matsui T, Sasaki H (1999) Oral care and pneumonia. Oral Care Working Group. Lancet 354:515

13. Kaklamanos EG, Charalampidou M, Menexes G, Topitsoglou V, Kalfas S (2005) Transient oral microflora in Greeks attending day centres for the elderly and residents in homes for the elderly. Gerodontology 22:158-167

14. Budtz-Jorgensen E, Rentsch A, Borgis S, Mojon P (1999) Oral health problems in the elderly. In: Michel JP, Hof PR (eds) Management of aging. Karger, Basel

15. Budtz-Jorgensen E (1999) Prosthodontics for the elderly: diagnosis and treatment. Quintessence Publishing Co, Inc, Chicago

16. Dodd MJ, Larson PJ, Dibble SL, Miaskowski C, Greenspan D, MacPhail L, Hauck WW, Paul SM, Ignoffo R, Shiba G (1996) Randomized clinical trial of chlorhexidine versus placebo for prevention of oral mucositis in patients receiving chemotherapy. Oncol Nurs Forum 23:921-927

17. Hagedorn B, Willershausen B, Ernst CP, Wehse T, Schimmel M (2000) A comparative study of 2 fluoride-based mouthrinses. Quintessence Int 31:125-128

18. Prabuseenivasan S, Jayakumar M, Ignacimuthu S (2006) In vitro activity of some plant essential oils. BMC Complement Altern Med $6: 1-8$

19. Schelz Z, Molnar J, Hohmann J (2006) Antimicrobial and antiplasmid activities of essential oils. Fitoterapia 77:279-285. https://doi.org/10.1016/j.fitote.2006.03.013

20. Sookto T, Srithavaj T, Thaweboon S, Thaweboon B, Shrestha B (2013) In vitro effects of Salvia officinalis L. essential oil on Candida albicans. Asian Pac J Trop Biomed 3:376-380

21. Delamare A, Moschen-Pistorello I, Artico L, Atti-Serafini L, Echeverrigaray S (2007) Antibacterial activity of the essential oils of Salvia officinalis L. and Salvia triloba L. cultivated in South Brazil. Food Chem 100:603-608

22. Hopewell S, Clarke M, Moher D, Wager E, Middleton P, Altman DG, Schulz KF (2008) CONSORT for reporting randomised trials in journal and conference abstracts. Lancet 371:281-283. https:// doi.org/10.1016/S0140-6736(07)61835-2

23. Ainamo J, Bay I (1975) Problems and proposals for recording gingivitis and plaque. Int Dent J 25:229-235

24. Lang NP, Joss A, Orsanic T, Gusberti FA, Siegrist BE (1986) Bleeding on probing. A predictor for the progression of periodontal disease? J Clin Periodontol 13:590-596

25. Loe H, Silness J (1963) Periodontal disease in pregnancy. I. Prevalence and severity. Acta Odontol Scand 21:533-551

26. Thomson WM, van der Putten GJ, de Baat C, Ikebe K, Matsuda K, Enoki K, Hopcraft MS, Ling GY (2011) Shortening the xerostomia inventory. Oral Surg Oral Med Oral Pathol Oral Radiol Endod 112: 322-327. https://doi.org/10.1016/j.tripleo.2011.03.024

27. Newton A (1962) Denture sore mouth. Br Dent J 112:357-359

28. The R Foundation (2019) The R Project for statistical computing. https://www.r-project.org.

29. Cronbach LJ (1951) Coefficient alpha and the internal structure of tests. Psychometrika 16:297-334

30. Bland JM, Altman DG (1997) Cronbach's alpha. BMJ 314:572
31. Clark LA, Watson D (1995) Constructing validity: basic issues in objective scale development. Psychol Assess 7:309-319

32. Sullivan GM, Feinn R (2012) Using effect size-or why the P value is not enough. J Grad Med Educ 4:279-282. https://doi.org/10. 4300/JGME-D-12-00156.1

33. Narayanan N, Thangavelu L (2015) Salvia officinalis in dentistry. Dent Hypotheses 6:27-30

34. Chalhoub E, Emami E, Freije M, Kandelman D, Campese M, StGeorges A, Voyer R, Rompre P, Barbeau J, Leduc A, Durand R (2016) Effectiveness of an alcohol-free essential oil-containing mouthwash in institutionalised elders receiving long-term care: a feasibility study. Gerodontology 33:69-78. https://doi.org/10. 1111/ger.12119

35. Sissons CH, Wong L, Cutress TW (1996) Inhibition by ethanol of the growth of biofilm and dispersed microcosm dental plaques. Arch Oral Biol 41:27-34

36. Marchetti E, Casalena F, Capestro A, Tecco S, Mattei A, Marzo G (2017) Efficacy of two mouthwashes on 3-day supragingival plaque regrowth: a randomized crossover clinical trial. Int J Dent Hyg 15: 73-80. https://doi.org/10.1111/idh.12185

37. Van Leeuwen M, Slot D, Van der Weijden G (2014) The effect of an essential-oils mouthrinse as compared to a vehicle solution on plaque and gingival inflammation: a systematic review and metaanalysis. Int J Dent Hyg 12:160-167

38. McCarney R, Warner J, Iliffe S, van Haselen R, Griffin M, Fisher P (2007) The Hawthorne effect: a randomised, controlled trial. BMC Med Res Methodol 7:30. https://doi.org/10.1186/1471-2288-7-30

39. McCambridge J, Witton J, Elbourne DR (2014) Systematic review of the Hawthorne effect: new concepts are needed to study research participation effects. J Clin Epidemiol 67:267-277. https://doi.org/ 10.1016/j.jclinepi.2013.08.015

40. Bellomo F, de Preux F, Chung JP, Julien N, Budtz-Jorgensen E, Muller F (2005) The advantages of occupational therapy in oral hygiene measures for institutionalised elderly adults. Gerodontology 22:24-31

41. Calderon-Montano JM, Jimenez-Alonso JJ, Guillen-Mancina E, Burgos-Moron E, Lopez-Lazaro M (2018) A 30-s exposure to ethanol $20 \%$ is cytotoxic to human keratinocytes: possible mechanistic link between alcohol-containing mouthwashes and oral cancer. Clin Oral Investig 22:2943-2946. https://doi.org/10.1007/s00784-0182602-z

42. Boffetta P, Hayes RB, Sartori S, Lee YC, Muscat J, Olshan A, Winn DM, Castellsague X, Zhang ZF, Morgenstern H, Chen C, SchwartZ SM, Vaughan TL, Wunsch-Filho V, Purdue M, Koifman S, Curado MP, Vilensky M, Gillison M, Fernandez L, Menezes A, Daudt AW, Schantz S, Yu G, D’Souza G, Haddad RI, La Vecchia C, Hashibe M (2016) Mouthwash use and cancer of the head and neck: a pooled analysis from the international head and neck Cancer epidemiology consortium. Eur J Cancer Prev 25:344-348. https://doi.org/10. 1097/cej.0000000000000179

43. Lachenmeier DW, Monakhova YB, Markova M, Kuballa T, Rehm J (2013) What happens if people start drinking mouthwash as surrogate alcohol? A quantitative risk assessment. Food Chem Toxicol 51:173-178. https://doi.org/10.1016/j.fct.2012.09.031

44. Nair R, Chiu SE, Chua YK, Dhillon IK, Li J, Yee Ting Fai R (2018) Should short-term use of alcohol-containing mouthrinse be avoided for fear of worsening xerostomia? J Oral Rehabil 45:140-146. https://doi.org/10.1111/joor.12587

Publisher's note Springer Nature remains neutral with regard to jurisdictional claims in published maps and institutional affiliations. 